\title{
Communiquer, former, informer en oncologie en 2018
}

\section{Communication, Training and Information in Oncology en 2018}

\author{
C. Ferrari - Responsable du département parcours de soins de l'Institut national du cancer (INCa) \\ (C) Lavoisier SAS 2018
}

L'Institut national du cancer a eu l'honneur cette année de faire le discours d'ouverture du XXXIV ${ }^{e}$ Congrès de la Société Française de Psycho-Oncologie dont la thématique centrale était la communication ; celle-ci se déclinait en plusieurs axes : la communication entre professionnels soignants, entre les médecins et les patients en incluant les proches, mais également la communication au niveau intrafamiliale. Toutes des composantes essentielles dans les soins des patients.

Parmi les thématiques abordées, il y eut celle de l'enseignement à la communication pour les jeunes médecins. Cette approche est en effet un des objectifs du troisième Plan cancer qui visait l'introduction de cet enseignement dans la maquette du diplôme d'études spécialisées en oncologie. Cette nouvelle maquette prévoit désormais l'amélioration des connaissances sur la relation médecin-patient atteint de cancer ainsi que le développement de compétences spécifiques à la spécialité comme, par exemple, savoir interroger, informer et communiquer avec un patient atteint de cancer. Ces maquettes sont entrées en vigueur en novembre 2017. Si ces nouveaux contenus permettent une sensibilisation à la problématique de la communication, ils ne sont que le premier pas vers une formation qui ne peut se faire que tout au long de la vie professionnelle. Ce XXXIV ${ }^{\mathrm{e}}$ Congrès de par les thématiques abordées en est une illustration.

Depuis le premier Plan cancer, les soins psychiques des patients atteints d'un cancer occupent une place importante. Des actions concrètes visant le soutien psychologique et plus globalement l'accompagnement du patient tout au long de son parcours de soins et dans l'après-cancer ont été soute- nues ; d'abord en promouvant la création de postes de psycho-oncologues au sein des établissements autorisés aux traitements du cancer, mais également en soutenant, par le biais d'appels à projets, diverses approches dans l'accompagnement des patients en souffrance psychique.

En 2016, l'INCa a publié un rapport sur les soins de support en cancérologie. Dans ce travail, l'Institut fait des recommandations de mise en œuvre du soutien psychologique des patients, des proches et des aidants et recommande le développement de la communication, de l'information et de la formation des aidants. Il met en outre l'accent sur la détection précoce et l'orientation des patients vers les professionnels ou les équipes les plus à même de les prendre en charge pour éviter toute perte de chance.

Une première déclinaison pratique de ce rapport a été réalisée en 2016. Il s'agit du Référentiel organisationnel pour le repérage et le traitement précoce de la souffrance psychique des patients atteints de cancer. L'objectif de ce référentiel est d'apporter aux professionnels de santé, où qu'ils pratiquent, des repères pour pouvoir détecter précocement la souffrance psychique et donner aux patients une réponse adaptée à leurs besoins. Cela implique une évaluation systématique tout au long de leur parcours de soins. Ce référentiel, qui a été publié au cours du premier trimestre 2018, s'adresse également aux patients et à leur entourage, car, nous le savons tous, celui-ci joue un rôle majeur auprès du patient.

Les diverses interventions et les ateliers pendant les trois jours du congrès ont permis d'aborder tous ces sujets avec des échanges très riches avec l'audience.

C. Ferrari $(\bowtie)$

52 , avenue André-Morizet,

F-92100 Boulogne-Billancourt, France

e-mail : cferrari@institutcancer.fr 